\title{
The Effect of Ion-Channel Guiding on the Chaotic Electron Trajectories in a Free Electron Laser
}

\author{
S. SAVIZ* AND H. MEHDIAN \\ Department of Physics, Teacher Training University, Tehran, Iran
}

(Received February 14, 2005)

\begin{abstract}
It has been confirmed that when the equilibrium self-fields were taken into account, the motion of an electron in a helical wiggler with axial magnetic field may be chaotic. In this paper, the effect of an ion-channel guiding on the chaotic electron trajectories is analyzed. It is shown that the ion-channel guiding may modify the chaotic electron trajectories. It is also found that when the parameter $\varepsilon$, i.e. $\varepsilon=\omega_{\mathrm{pb}}^{2} / 4 \omega_{\mathrm{c}}^{2}$, is increased, the density of the ion-channel guiding, i.e. $\alpha_{\mathrm{i}}=\omega_{\mathrm{pi}}^{2} / 4 \omega_{\mathrm{c}}^{2}$, must be increased to keep the modification of the chaotic motion. The chaotic trajectories for constant parameter $\varepsilon$ and increased density of the ion-channel guiding are presented. Numerical calculations are used to find the intersection between steady state group I and group II and resonance curves. Poincaré surface-ofsection maps are generated to demonstrate the chaotic electron trajectories with axial magnetic field and its modification in the presence of an ion-channel guiding.
\end{abstract}

PACS numbers: 41.60.Cr

\section{Introduction}

The free electron laser (FEL) makes use of the unstable interaction of a relativistic electron beam with a transverse wiggler magnetic field to generate coherent electromagnetic radiation $[1,2]$.

A uniform static axial magnetic field generated by the current in a solenoid is often employed to collimate the intense electron beam in the transverse direction. Ion-channel guiding of the relativistic electron beam in a FEL has been employed

*corresponding author; e-mail: s_saviz@tmu.ac.ir 
as an alternative to guiding by axial magnetic field $[3,4]$. Recent study of the electron trajectories in a helical wiggler and planner with ion-channel guiding has been carried out $[5,6]$.

Although there have been a great deal of studies on the theory of the FEL, most treatments have neglected the influence of the equilibrium self-fields [7] of the (non-neutral) electron beam, while such an approximation is valid for low current FEL operation. It was shown that equilibrium self-field effects play a significant role in altering the electron dynamics when the beam current $I_{\mathrm{b}}$ approaches the multi kiloamper range [8]. The analysis of self-field induced chaoticity in the electron orbits in a helical wiggler FEL with axial guide field have been carried out [1]. In this paper, the motion of a relativistic electron in the field configuration consisting of a constant amplitude helical wiggler magnetic field $\boldsymbol{B}_{\mathrm{w}}(\boldsymbol{x})$, a uniform axial magnetic field $\boldsymbol{B}_{0} \hat{e}_{z}$, the equilibrium self-electric and magnetic fields and an ion-channel guiding have been analyzed. A uniform density non-neutral beam produced the equilibrium self-electric and magnetic fields. Moreover, a positive ion core formed by expulsion of electrons from a preionized plasma channel into which the electron beam was injected formed the ion-channel guiding. It is shown that the motion is integrable. Poincaré surface-of-section maps are generated to demonstrate the integrability of the motion. As the dimensionless equilibrium self-field parameter $\varepsilon=\omega_{\mathrm{pb}}^{2} / 4 \omega_{\mathrm{c}}^{2}$ is increased in size, the dimensionless of the ion-channel guiding parameter must be increased to keep the integrability of the motion. [Here $\omega_{\mathrm{pb}}^{2}=4 \pi e^{2} n_{\mathrm{b}} / m$ and $\omega_{\mathrm{c}}=e B_{0} / m c$ are the non-relativistic plasma and cyclotron frequencies, respectively.] Poincaré surface-of-section maps are drawn to demonstrate the nonintegrability and chaoticity of the motion when the self-field parameter $\varepsilon$ was fixed, but the ion-channel guiding parameter $\alpha_{\mathrm{i}}$ was increased. In Sect. 2 the dynamical problem is formulated in canonical variables, and the constant of the motion is determined by means of canonical transformations. In Sect. 3 the (integrable) motion of an electron in the wiggler and guide field is given or obtained. The (nonintegrable) electron trajectories due to the effects of equilibrium self-fields are determined and then the effects of an ion-channel guiding on the chaotic motion arisen by the equilibrium self-fields are analyzed. In Sect. 4, by generating the Poincaré surface-of-section maps, we see chaotic electron trajectories without ion-channel guiding and its modification in the presence of the ion-channel guiding.

\section{Theoretical formulation of the problem}

Let us consider the motion of a relativistic electron beam in a uniform magnetic field $\boldsymbol{B}=B_{0} \hat{\boldsymbol{e}}_{z}$, a constant amplitude helical wiggler magnetic field $\boldsymbol{B}_{\mathrm{w}}=-B_{\mathrm{w}}\left(\hat{\boldsymbol{e}}_{x} \cos k_{\mathrm{w}} z+\hat{\boldsymbol{e}}_{y} \sin k_{\mathrm{w}} z\right)$, self-electric and magnetic fields produced by relativistic non-neutral electron beam with radius $r_{\mathrm{b}}$, average axial velocity $V_{\mathrm{b}} \hat{\boldsymbol{e}}_{z}$ and uniform density profile 


$$
n_{\mathrm{b}}^{0}= \begin{cases}n_{\mathrm{b}}=\text { const }, & 0 \leq r \leq r_{\mathrm{b}} \\ 0, & r>r_{\mathrm{b}}\end{cases}
$$

where $r_{\mathrm{b}}=\left(x^{2}+y^{2}\right)^{1 / 2}$ is the radial distance from the beam center, it follows from the steady-state Maxwell equations that the $\boldsymbol{E}_{\mathrm{s}}$ and $\boldsymbol{B}_{\mathrm{s}}$ can be expressed as

$$
\boldsymbol{E}_{\mathrm{s}}=-\frac{m_{\mathrm{e}} \omega_{\mathrm{pb}}^{2}}{2 e}\left(x \hat{\boldsymbol{e}}_{x}+y \hat{\boldsymbol{e}}_{y}\right)
$$

and

$$
\boldsymbol{B}_{\mathrm{s}}=\frac{\omega_{\mathrm{pb}}^{2} \beta_{\mathrm{b}}}{2 e}\left(y \hat{\boldsymbol{e}}_{x}-x \hat{\boldsymbol{e}}_{y}\right) .
$$

Here $\beta_{\mathrm{b}}=V_{\mathrm{b}} / c$ is the normalized beam velocity and $\omega_{\mathrm{pb}}=\left(4 \pi e^{2} n_{\mathrm{b}} / m_{\mathrm{e}}\right)^{1 / 2}$ is the non-relativistic plasma frequency of the beam electrons.

The transverse electrostatic field generated by an ion-channel guiding can be written as [3]:

$$
\boldsymbol{E}_{\mathrm{i}}=2 \pi e n_{\mathrm{i}}\left(x \hat{\boldsymbol{e}}_{x}+y \hat{\boldsymbol{e}}_{y}\right),
$$

where $n_{\mathrm{i}}$ is the charge density of the ion-channel guiding.

In the present analysis diamagnetic and paramagnetic effects [9] are neglected. The equation of motion for an electron within the beam $\left(0 \leq r \leq r_{\mathrm{b}}\right)$ can be derived from the Hamiltonian

$$
H=\left[(\boldsymbol{P} c+e \boldsymbol{A})^{2}+m^{2} c^{4}\right]^{1 / 2}-e \phi_{\mathrm{i}}-e \phi_{\mathrm{s}} \equiv \gamma m c^{2}-e \phi_{\mathrm{i}}-e \phi_{\mathrm{s}},
$$

where $\gamma=\left[1+\left(p / m c^{2}\right)\right]^{1 / 2}$ is the relativistic mass factor and the scalar potentials $\phi_{\mathrm{i}}, \phi_{\mathrm{s}}$, and the vector potential $\boldsymbol{A}$ can be written as

$$
\begin{aligned}
& \phi_{\mathrm{i}}=-\frac{m_{\mathrm{i}} \omega_{\mathrm{pi}}^{2}}{4 e}\left(x^{2}+y^{2}\right), \\
& \phi_{\mathrm{s}}=\frac{m_{\mathrm{e}} \omega_{\mathrm{pb}}^{2}}{4 e}\left(x^{2}+y^{2}\right), \\
& \boldsymbol{A}=B_{0} x \hat{\boldsymbol{e}}_{y}+A_{\mathrm{w}}\left(\hat{\boldsymbol{e}}_{x} \cos k_{\mathrm{w}} z+\hat{\boldsymbol{e}}_{y} \sin k_{\mathrm{w}} z\right)+\beta_{\mathrm{b}} \phi_{\mathrm{s}} \hat{\boldsymbol{e}}_{\mathrm{z}},
\end{aligned}
$$

where $A_{\mathrm{w}}=B_{\mathrm{w}} / k_{\mathrm{w}}=$ const, and $\omega_{\mathrm{pi}}^{2}=\left(4 \pi e^{2} n_{\mathrm{i}} / m_{\mathrm{i}}\right)^{1 / 2}$ is the non-relativistic plasma frequency of the ion channel. The mechanical momentum $\boldsymbol{p}$ is related to the canonical momentum $\boldsymbol{P}$ by $\boldsymbol{p}=\boldsymbol{P}+e \boldsymbol{A} / c$. Because $H$ is independent of time, the Hamiltonian is a constant of motion

$$
H=\gamma m c^{2}-e \phi_{\mathrm{i}}-e \phi_{\mathrm{s}} \equiv \text { const }
$$

corresponding to the conservation of the total energy of an individual electron.

In order to find an additional constant of the motion and to determine the resonance, we use the canonical transformation [1]. The transformation in new variables $\left(\phi, \psi, z^{\prime}, P_{\phi}, P_{\psi}, P_{z^{\prime}}\right)$ is 


$$
\begin{aligned}
& x=\left(\frac{2 P_{\phi}}{m \omega_{\mathrm{c}}}\right)^{1 / 2} \sin \left(\varphi+k_{\mathrm{w}} z^{\prime}\right)-\left(\frac{2 P_{\psi}}{m \omega_{\mathrm{c}}}\right)^{1 / 2} \cos \left(\psi-k_{\mathrm{w}} z^{\prime}\right), \\
& y=\left(\frac{2 P_{\psi}}{m \omega_{\mathrm{c}}}\right)^{1 / 2} \sin \left(\psi-k_{\mathrm{w}} z^{\prime}\right)-\left(\frac{2 P_{\varphi}}{m \omega_{\mathrm{c}}}\right)^{1 / 2} \cos \left(\varphi+k_{\mathrm{w}} z^{\prime}\right), \\
& z=z^{\prime} \\
& P_{x}=\left(2 m \omega_{\mathrm{c}} P_{\varphi}\right)^{1 / 2} \cos \left(\varphi+k_{\mathrm{w}} z^{\prime}\right), \\
& P_{y}=\left(2 m \omega_{\mathrm{c}} P_{\psi}\right)^{1 / 2} \cos \left(\psi-k_{\mathrm{w}} z^{\prime}\right), \\
& P_{z}=P_{z^{\prime}}-k_{\mathrm{w}} P_{\varphi}+k_{\mathrm{w}} P_{\psi},
\end{aligned}
$$

where $\omega_{\mathrm{c}}=e B_{0} / m c$ is the cyclotron frequency of non-relativistic beam, the Hamiltonian in the new variables $\left(\varphi, \psi, P_{\varphi}, P_{\psi}, P_{z^{\prime}}, z^{\prime}\right)$ is given by

$$
\begin{aligned}
\frac{1}{m c^{2}} & H\left(\varphi, \psi, P_{\varphi}, P_{\psi}, P_{z^{\prime}}, z^{\prime}\right) \\
& =-\frac{e \phi_{\mathrm{s}}}{m c^{2}}-\frac{e \phi_{\mathrm{i}}}{m c^{2}}+\left[\frac{2 \omega_{\mathrm{c}} P_{\varphi}}{m c^{2}}+\frac{2 e A_{\mathrm{w}}}{m c^{2}}\left(\frac{2 \omega_{\mathrm{c}} P_{\varphi}}{m c^{2}}\right)^{1 / 2} \cos \varphi\right. \\
& \left.+\left(\frac{P_{z^{\prime}}-k_{\mathrm{w}} P_{\varphi}+k_{\mathrm{w}} P_{\psi}}{m c}+\beta_{\mathrm{b}} \frac{e \phi_{\mathrm{s}}}{m c^{2}}\right)^{2}+\left(\frac{e A_{\mathrm{w}}}{m c^{2}}\right)^{2}+1\right]^{1 / 2},
\end{aligned}
$$

where $\phi_{\mathrm{s}}, \varepsilon, \phi_{\mathrm{i}}$ and $\alpha_{\mathrm{i}}$ are defined by

$$
\begin{aligned}
& e \phi_{\mathrm{s}}=2 \varepsilon \omega_{\mathrm{c}}\left[P_{\varphi}+P_{\psi}-2\left(P_{\varphi} P_{\psi}\right)^{1 / 2} \sin (\varphi+\psi)\right], \\
& e \phi_{\mathrm{i}}=-2 \alpha_{\mathrm{i}} \omega_{\mathrm{c}}\left[P_{\varphi}+P_{\psi}-2\left(P_{\varphi} P_{\psi}\right)^{1 / 2} \sin (\varphi+\psi)\right], \\
& \varepsilon=\omega_{\mathrm{pb}}^{2} / 4 \omega_{\mathrm{c}}^{2}, \\
& \alpha_{\mathrm{i}}=\omega_{\mathrm{pi}}^{2} / 4 \omega_{\mathrm{c}}^{2} .
\end{aligned}
$$

The potentials $\phi_{\mathrm{s}}$ and $\phi_{\mathrm{i}}$ in the new variables are obtained by substituting Eqs. (10)-(15) into Eqs. (6), (7). The dimensionless parameters $\varepsilon$ and $\alpha_{\mathrm{i}}$ characterize the strength of the equilibrium self-fields and ion-channel guiding. The dimensionless parameters and variables defined by

$$
\begin{aligned}
& \hat{\omega}_{\mathrm{c}}=\frac{\omega_{\mathrm{c}}}{c k_{\mathrm{w}}}, \quad \hat{\omega}_{\mathrm{pb}}=\frac{\omega_{\mathrm{pb}}}{c k_{\mathrm{w}}}, \quad \hat{\omega}_{\mathrm{pi}}=\frac{\omega_{\mathrm{pi}}}{c k_{\mathrm{w}}}, \quad \hat{\phi}_{\mathrm{s}}=\frac{e \phi_{\mathrm{s}}}{m c^{2}}, \quad \hat{\phi}_{\mathrm{i}}=\frac{e \phi_{\mathrm{i}}}{m c^{2}}, \\
& a_{\mathrm{w}}=\frac{e A_{\mathrm{w}}}{m c^{2}}, \quad \hat{P}_{z}=\frac{P_{z}}{m c}, \quad \hat{P}_{\varphi}=\frac{k_{\mathrm{w}} P_{\varphi}}{m c}, \quad \hat{P}_{\psi}=\frac{k_{\mathrm{w}} P_{\psi}}{m c}, \quad \hat{H}=\frac{H}{m c^{2}}, \\
& \tau=c k_{\mathrm{w}} t, \quad \hat{z}^{\prime}=k_{\mathrm{w}} z^{\prime},
\end{aligned}
$$

have been introduced. 
Therefore the dimensionless of $H, \phi_{\mathrm{s}}$ and $\phi_{\mathrm{i}}$ defined in Eqs. (16), (17) and (18) respectively, becomes

$$
\begin{aligned}
& \hat{H}\left(\varphi, \hat{\psi}, \hat{P}_{\varphi}, \hat{P}_{\psi}, \hat{P}_{z^{\prime}}\right)=-\hat{\phi}_{\mathrm{s}}-\hat{\phi}_{\mathrm{i}}+\left[2 \hat{\omega}_{\mathrm{c}} \hat{P}_{\varphi}+2 a_{\mathrm{w}}\left(2 \hat{\omega}_{\mathrm{c}} \hat{P}_{\varphi}\right)^{1 / 2} \cos \varphi\right. \\
& \left.\quad+\left(\hat{P}_{z^{\prime}}-\hat{P}_{\varphi}+\hat{P}_{\psi}+\beta_{\mathrm{b}} \hat{\phi}_{\mathrm{s}}\right)^{2}+a_{\mathrm{w}}^{2}+1\right]^{1 / 2}, \\
& \hat{\phi}_{\mathrm{s}}=2 \varepsilon \hat{\omega}_{\mathrm{c}}\left(\hat{P}_{\varphi}+\hat{P}_{\psi}-2\left(\hat{P}_{\varphi} \hat{P}_{\psi}\right)^{1 / 2} \sin (\varphi+\psi)\right], \\
& \hat{\phi}_{\mathrm{i}}=-2 \alpha_{\mathrm{i}} \hat{\omega}_{\mathrm{c}}\left(\hat{P}_{\varphi}+\hat{P}_{\psi}-2\left(\hat{P}_{\varphi} \hat{P}_{\psi}\right)^{1 / 2} \sin (\varphi+\psi)\right] .
\end{aligned}
$$

Since $\hat{H}$ is independent of $\hat{z}^{\prime}$, it follows that $\hat{P}_{z^{\prime}}$ is a constant of motion.

\section{Equations of motion}

In the Hamiltonian of Eq. (22), setting $\varepsilon$ and $\alpha_{\mathrm{i}}$ equal to zero, the effects of equilibrium self-field and an ion-channel guiding contribution vanish $\left(\hat{\phi}_{\mathrm{s}}=0\right.$, $\left.\hat{\phi}_{\mathrm{i}}=0\right)$. Therefore the Hamiltonian in Eq. (22) reduces to

$$
\begin{aligned}
& \hat{H}_{0}\left(\varphi, \hat{P}_{\varphi}, \hat{P}_{\psi}, \hat{P}_{z^{\prime}}\right)=\left[2 \hat{\omega}_{\mathrm{c}} \hat{P}_{\varphi}+2 a_{\mathrm{w}}\left(2 \hat{\omega}_{\mathrm{c}} \hat{P}_{\varphi}\right)^{1 / 2} \cos \varphi\right. \\
& \left.+\left(\hat{P}_{z^{\prime}}-\hat{P}_{\varphi}+\hat{P}_{\psi}\right)^{2}+a_{\mathrm{w}}^{2}+1\right]^{1 / 2} \equiv \gamma_{0} .
\end{aligned}
$$

The motion described by Eq. (25) is integrable and has been analyzed by several authors [10]. Equations of motion for fixed points or steady state orbits denoted by $\varphi_{0}, \hat{P}_{\varphi_{0}}$ and the equation of $\hat{P}_{z_{0}}$ for locating the fixed points in a given electron energy $\gamma_{0}$ have been obtained by [1].

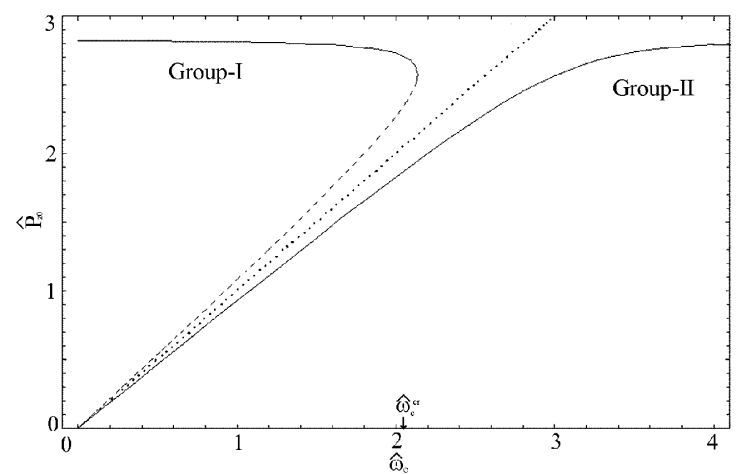

Fig. 1. Graph of the integrable steady-state trajectories calculated from Eq. (37) for $\varepsilon=0, \gamma_{0}=3$ and $a_{\mathrm{w}}=0.2$. The solid (dashed) curves correspond to the stable (unstable) orbits, and the dotted straight line designates the magnetoresonance condition $\hat{p}_{z}=\hat{\omega}_{\mathrm{c}}$.

Figure 1 shows the typical magnetic field dependence where dotted straight line represents magnetoresonance. The chaotic motion for self-fields for $\varepsilon \neq 0$ and 
without ion-channel guiding was given in the paper of Chen and Davidson [8]. Here we obtain the equation of electron trajectories with $\varepsilon \neq 0$ and $\alpha_{\mathrm{i}} \neq 0$ (self-field and ion-channel guiding). From the Hamiltonian defined in Eq. (22), we obtain

$$
\begin{aligned}
& \frac{\mathrm{d} \varphi}{\mathrm{d} \tau}=\frac{\partial \hat{H}}{\partial \hat{P}_{\varphi}}=-\frac{\hat{p}_{z}}{\gamma}+\frac{\hat{\omega}_{\mathrm{c}}}{\gamma}\left[1+\frac{a_{\mathrm{w}}}{\left(2 \hat{\omega}_{\mathrm{c}} \hat{P}_{\varphi}\right)^{1 / 2}} \cos \varphi\right] \\
& -2 \varepsilon \hat{\omega}_{\mathrm{c}}\left(1-\beta_{\mathrm{b}} \frac{\hat{p}_{z}}{\gamma}\right)\left[1-\left(\frac{\hat{P}_{\psi}}{\hat{P}_{\varphi}}\right)^{1 / 2} \sin (\varphi+\psi)\right] \\
& +2 \alpha_{\mathrm{i}} \hat{\omega}_{\mathrm{c}}\left[1-\left(\frac{\hat{P}_{\psi}}{\hat{P}_{\varphi}}\right)^{1 / 2} \sin (\varphi+\psi)\right] \\
& \frac{\mathrm{d} \psi}{\mathrm{d} \tau}=\frac{\partial \hat{H}}{\partial \hat{P}_{\varphi}}=\frac{\hat{p}_{z}}{\gamma}-2 \varepsilon \hat{\omega}_{\mathrm{c}}\left(1-\beta_{\mathrm{b}} \frac{\hat{p}_{z}}{\gamma}\right)\left[1-\left(\frac{\hat{P}_{\varphi}}{\hat{P}_{\psi}}\right)^{1 / 2} \sin (\varphi+\psi)\right] \\
& +2 \alpha_{\mathrm{i}} \hat{\omega}_{\mathrm{c}}\left[1-\left(\frac{\hat{P}_{\varphi}}{\hat{P}_{\psi}}\right)^{1 / 2} \sin (\varphi+\psi)\right] \\
& \frac{\mathrm{d} \hat{P}_{\varphi}}{\mathrm{d} \tau}=-\frac{\partial \hat{H}}{\partial \varphi}=\frac{a_{\mathrm{w}}}{\gamma}\left(2 \hat{\omega}_{\mathrm{c}} \hat{P}_{\varphi}\right)^{1 / 2} \cos \varphi \\
& -4 \varepsilon \hat{\omega}_{\mathrm{c}}\left(1-\frac{\beta_{\mathrm{b}} \hat{p}_{z}}{\gamma}\right)\left(\hat{P}_{\varphi} \hat{P}_{\psi}\right)^{1 / 2} \cos (\varphi+\psi) \\
& +4 \alpha_{\mathrm{i}} \hat{\omega}_{\mathrm{c}}\left(\hat{P}_{\varphi} \hat{P}_{\psi}\right)^{1 / 2} \cos (\varphi+\psi), \\
& \frac{\mathrm{d} \hat{P}_{\psi}}{\mathrm{d} \tau}=-\frac{\partial \hat{H}}{\partial \psi}=-4 \varepsilon \hat{\omega}_{\mathrm{c}}\left(1-\frac{\beta_{\mathrm{b}} \hat{p}_{z}}{\gamma}\right)\left(\hat{P}_{\varphi} \hat{P}_{\psi}\right)^{1 / 2} \cos (\varphi+\psi) \\
& +4 \alpha_{\mathrm{i}} \hat{\omega}_{\mathrm{c}}\left(\hat{P}_{\varphi} \hat{P}_{\psi}\right)^{1 / 2} \cos (\varphi+\psi),
\end{aligned}
$$

where the normalized axial mechanical momentum $\hat{p}_{z}=\hat{P}_{z^{\prime}}-\hat{P}_{\varphi}+\hat{P}_{\psi}+\beta_{\mathrm{b}} \hat{\phi}_{\mathrm{s}}$ has been introduced for simplicity of notation. Let us note that the constant of $\hat{H}_{0}$ in Eq. (25) is now replaced by $\hat{H}=\gamma-\hat{\phi}_{\mathrm{s}}-\hat{\phi}_{\mathrm{i}}$, where $\hat{P}_{\psi}$ is no longer a constant of motion.

To provide a semiquantitative understanding of the motion near the group-I and group-II orbits, the first order, self-field induced resonance is analyzed as follows. For $\varepsilon \ll 1$, the Hamiltonian in Eq. (22), by considering the first order of $\varepsilon$, can be expanded as 


$$
\hat{H}=\hat{H}_{0}+\hat{H}_{1},
$$

where $\hat{H}_{0}=\gamma_{0}$ and $\hat{H}_{1}$ can be expanded as

$$
\hat{H}_{1}=2 \hat{\omega}_{\mathrm{c}}\left[\varepsilon\left(\beta_{\mathrm{b}} \frac{\hat{p}_{z}}{\gamma_{0}}-1\right)+\alpha_{\mathrm{i}}\right]\left[\hat{P}_{\varphi}+\hat{P}_{\psi}-2\left(\hat{P}_{\varphi} \hat{P}_{\psi}\right)^{1 / 2} \sin (\varphi+\psi)\right] .
$$

It was shown that the zero-order Hamiltonian $H_{0}=\gamma_{0}$ is integrable, see Fig. 2a and $\mathrm{b}$. For an electron oscillating about an integrable stable steady-state orbit described by $\varphi(\tau)=\varphi_{0}+\delta \varphi \cos \hat{\omega} \tau$ and $\hat{P}_{\varphi}=\hat{P}_{\varphi_{0}}+\delta \hat{P}_{\varphi} \sin \hat{\omega} \tau$, the perturbation $\hat{H}_{1}$ can be approximated by

$$
\begin{aligned}
\hat{H}_{1}= & 2 \hat{\omega}_{\mathrm{c}}\left[\varepsilon\left(\beta_{\mathrm{b}}^{2}-1\right)+\alpha_{\mathrm{i}}\right] \\
& \times\left[\hat{P}_{\varphi_{0}}+\hat{P}_{\psi_{0}}-2\left(\hat{P}_{\varphi_{0}} \hat{P}_{\psi_{0}}\right)^{1 / 2} \sin \left(\varphi_{0}+\delta \varphi_{0} \cos \omega \tau+\psi\right)\right] \\
& =2 \hat{\omega}_{\mathrm{c}}\left[\varepsilon\left(\beta_{\mathrm{b}}^{2}-1\right)+\alpha_{\mathrm{i}}\right]\left\{\hat{P}_{\varphi_{0}}+\hat{P}_{\psi_{0}}-2\left(\hat{P}_{\varphi_{0}} \hat{P}_{\psi_{0}}\right)^{1 / 2}\right. \\
& \left.\times \sum_{n=-\infty}^{\infty} J_{n}\left(\delta \varphi_{0}\right) \sin \left[\left(\hat{\omega} \tau+\frac{\pi}{2}\right) n+\psi+\varphi_{0}\right]\right\} .
\end{aligned}
$$

Here $\hat{\omega}$ is the normalized oscillation frequency for both group I and group II defined by $[8]$ :

$$
\hat{\omega}=\frac{\left|\hat{p}_{z_{0}}-\hat{\omega}_{\mathrm{c}}\right|}{\gamma_{0}}\left(1-\frac{\hat{\omega}_{\mathrm{c}} a^{2}{ }_{\mathrm{w}}}{\left(\hat{p}_{z_{0}}-\hat{\omega}_{\mathrm{c}}\right)^{3}}\right)^{1 / 2},
$$

where $J_{n}(x)$ is the Bessel function of the first kind of order one, and we have made approximation $\beta_{z_{0}} \cong \beta_{\mathrm{b}}$.
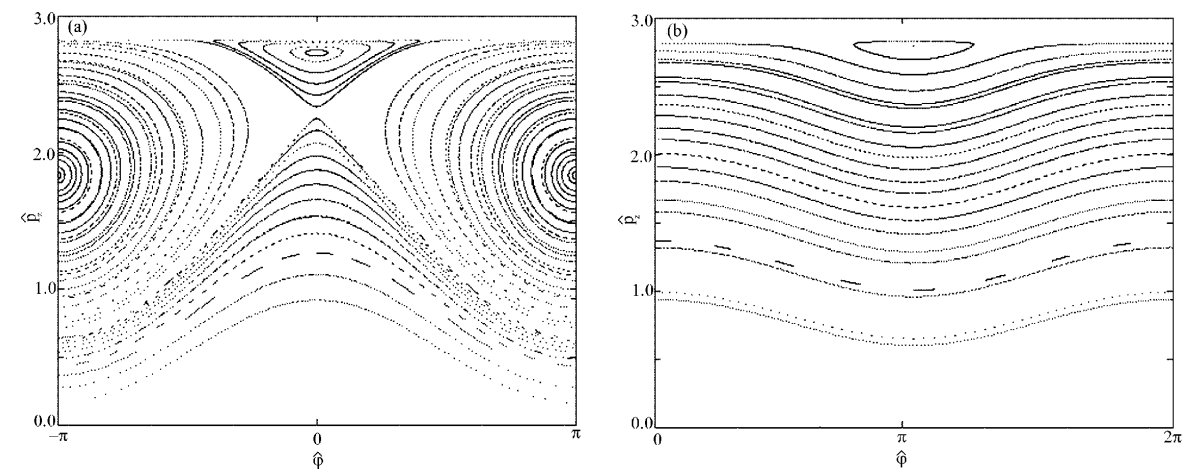

Fig. 2. Graph of the integrable phase plane $\left(\varphi, \hat{p}_{z}\right)$ calculated from Eq. (25) for $\varepsilon=0, \gamma_{0}=3$ and $a_{\mathrm{w}}=0.2$. The two cases correspond to (a) $\hat{\omega}_{\mathrm{c}}=2.0<\hat{\omega}_{\mathrm{cr}} \cong 2.1$ and (b) $\hat{\omega}=4.0>\hat{\omega}_{\mathrm{cr}} \cong 2.1$. 
It follows from Eq. (33) that the self-field induced resonance in presence of the ion-channel guiding is given by

$$
\frac{\mathrm{d} \psi}{\mathrm{d} \tau}+n \hat{\omega}=0
$$

Substituting Eq. (27) and Eq. (33) into Eq. (34) yields

$$
\begin{aligned}
\frac{\hat{p}_{z}}{\gamma}- & 2 \varepsilon \omega_{\mathrm{c}}\left(1-\beta_{\mathrm{b}} \frac{\hat{p}_{z}}{\gamma}\right)\left[1-\left(\frac{\hat{P}_{\varphi}}{\hat{P}_{\psi}}\right)^{1 / 2} \sin (\varphi+\psi)\right] \\
& +2 \alpha_{\mathrm{i}} \hat{\omega}_{\mathrm{c}}\left[1-\left(\frac{\hat{P}_{\varphi}}{\hat{P}_{\psi}}\right)^{1 / 2} \sin (\varphi+\psi)\right] \\
& +n \frac{\left|\hat{p}_{z_{0}}-\hat{\omega}_{\mathrm{c}}\right|}{\gamma_{0}}\left(1-\frac{\hat{\omega}_{\mathrm{c}} a_{\mathrm{w}}{ }^{2}}{\left(\hat{p}_{z_{0}}-\hat{\omega}_{\mathrm{c}}\right)^{3}}\right)^{1 / 2}=0 .
\end{aligned}
$$

Averaging over the oscillation period, $2 \pi / \hat{\omega}$, of Eq. (35), we obtain the resonance conditions,

$$
\begin{aligned}
\frac{\hat{p}_{z_{0}}}{\gamma_{0}} & -2 \varepsilon \hat{\omega}_{\mathrm{c}}\left(1-\beta_{\mathrm{b}} \frac{\hat{p}_{z_{0}}}{\gamma_{0}}\right)+2 \alpha_{\mathrm{i}} \hat{\omega}_{\mathrm{c}}+n \frac{\left|\hat{p}_{z_{0}}-\hat{\omega}_{\mathrm{c}}\right|}{\gamma_{0}}\left(1-\frac{\hat{\omega}_{\mathrm{c}} a_{\mathrm{w}}^{2}}{\left(\hat{p}_{z_{0}}-\hat{\omega}_{\mathrm{c}}\right)^{3}}\right)^{1 / 2} \\
& =0
\end{aligned}
$$

\section{Numerical results and discussion}

A numerical study of integrable steady-state orbits, for group I and group II, nonintegrable orbits arising by self-fields and modified chaotic electron trajectories generated by the effect of ion-channel guiding is presented.

In Fig. 1, the group-I and group-II orbits are shown. For drawing this figure we use the equation [8]:

$$
\hat{p}_{z_{0}}^{2}\left[1+\frac{a_{\mathrm{w}}{ }^{2}}{\left(\hat{p}_{z_{0}}-\hat{\omega}_{\mathrm{c}}\right)^{2}}\right]+1=\gamma_{0}^{2} .
$$

This equation has, at most, four real roots for $\hat{p}_{z_{0}}$. Of this solution, one corresponds to backwards propagation (i.e. $\hat{p}_{z_{0}}<0$ ), which will be ignored. The remaining steady-state solutions may be divided into two classes corresponding to the cases in which: $\hat{\omega}_{\mathrm{c}}<\hat{p}_{z_{0}}$ referred to group I, and $\hat{\omega}_{\mathrm{c}}>\hat{p}_{z_{0}}$ referred to group II.

In Fig. 2, the phase space structure of $\varepsilon=0, \alpha_{\mathrm{i}}=0$ for two cases $0<$ $\hat{\omega}_{\mathrm{c}}<\hat{\omega}_{\mathrm{cr}}$ and $\hat{\omega}>\hat{\omega}_{\mathrm{cr}}$ belongs to group I and group II, respectively. The elliptic fixed points corresponding to steady-state orbits and the hyperbolic fixed points belong to unstable steady-state orbits. The stable and unstable orbits of group I are illustrated in Fig. 2a and the phase space structure i.e. stable state, of group II are shown in Fig. 2b. As seen in Fig. 2b, its orbits appear in the elliptic form. 
In Eq. (22) by setting $\alpha_{\mathrm{i}}=0$ without ion-channel guiding, the equations of motion are obtained [1]:

$$
\begin{aligned}
& \frac{\mathrm{d} \varphi}{\mathrm{d} \tau}= \frac{\partial \hat{H}}{\partial \hat{P}_{\varphi}}=-\frac{\hat{p}_{z}}{\gamma}+\frac{\hat{\omega}_{\mathrm{c}}}{\gamma}\left[1+\frac{a_{\mathrm{w}}}{\left(2 \hat{\omega}_{\mathrm{c}} \hat{P}_{\varphi}\right)^{1 / 2}} \cos \varphi\right] \\
&-2 \varepsilon \hat{\omega}_{\mathrm{c}}\left(1-\beta_{\mathrm{b}} \frac{\hat{p}_{z}}{\gamma}\right)\left[1-\left(\frac{\hat{P}_{\psi}}{\hat{P}_{\varphi}}\right)^{1 / 2} \sin (\varphi+\psi)\right] \\
& \frac{\mathrm{d} \psi}{\mathrm{d} \tau}=\frac{\partial \hat{H}}{\partial \hat{P}_{\varphi}}=\frac{\hat{p}_{z}}{\gamma}-2 \varepsilon \hat{\omega}_{\mathrm{c}}\left(1-\beta_{\mathrm{b}} \frac{\hat{p}_{z}}{\gamma}\right)\left[1-\left(\frac{\hat{P}_{\varphi}}{\hat{P}_{\psi}}\right)^{1 / 2} \sin (\varphi+\psi)\right] \\
& \frac{\mathrm{d} \hat{P}_{\varphi}}{\mathrm{d} \tau}=-\frac{\partial \hat{H}}{\partial \varphi}=\frac{a_{\mathrm{w}}}{\gamma}\left(2 \hat{\omega}_{\mathrm{c}} \hat{P}_{\varphi}\right)^{1 / 2} \cos \varphi \\
&-4 \varepsilon \hat{\omega}_{\mathrm{c}}\left(1-\frac{\beta_{\mathrm{b}} \hat{p}_{z}}{\gamma}\right)\left(\hat{P}_{\varphi} \hat{P}_{\psi}\right)^{1 / 2} \cos (\varphi+\psi) \\
& \frac{\mathrm{d} \hat{P}_{\psi}}{\mathrm{d} \tau}=-\frac{\partial \hat{H}}{\partial \psi}=-4 \varepsilon \hat{\omega}_{\mathrm{c}}\left(1-\frac{\beta_{\mathrm{b}} \hat{p}_{z}}{\gamma}\right)\left(\hat{P}_{\varphi} \hat{P}_{\psi}\right)^{1 / 2} \cos (\varphi+\psi)
\end{aligned}
$$

Poincaré surface-of-section maps have been generated by numerically integrating the equation of motion expressed in Eqs. (26)-(29). This analysis demonstrated the chaotic motion. The motion described by Eqs. (26)-(29) occurs in the three-dimensional phase space $\left(\varphi, \psi, \hat{P}_{\psi}\right)$, because $\hat{P}_{\psi}$ is determined from the constant of $\hat{H}$ and $\hat{p}_{z}$. Figure 3 shows typical nonintegrable surface-of-section maps for two cases: (a) $0<\hat{\omega}_{\mathrm{c}}<\hat{\omega}_{\mathrm{cr}}$ and (b) $\hat{\omega}_{\mathrm{c}}>\hat{\omega}_{\mathrm{cr}}$.
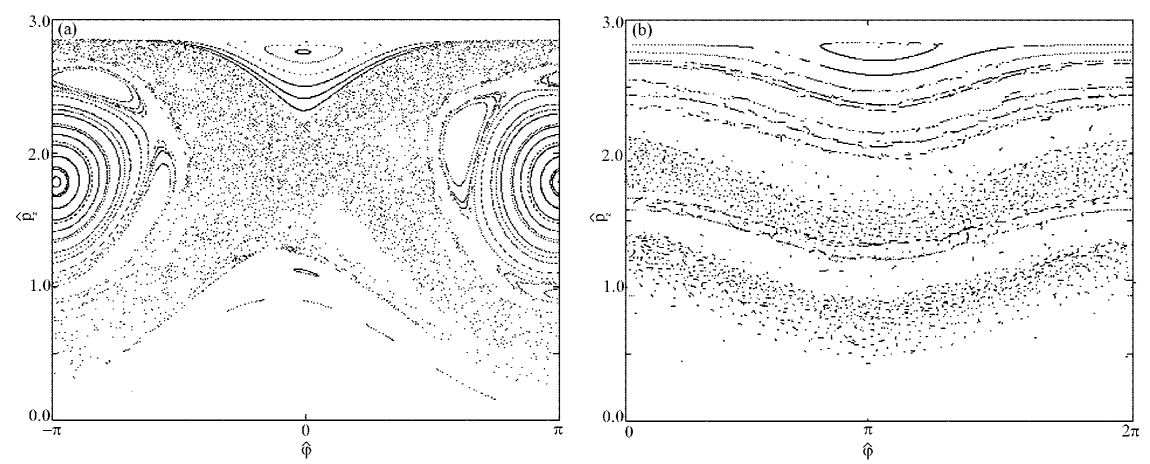

Fig. 3. Graph of nonintegrable surface-of-section for two cases: $\varepsilon=0.01, \hat{H}=3.0$, (a) $0<\hat{\omega}_{\mathrm{c}}=2.0<\hat{\omega}_{\mathrm{cr}} \cong 2.1$, (b) $\omega_{\mathrm{c}}=4.0>\omega_{\mathrm{cr}}$. Other system parameters are (a) $\varepsilon=0.01, \hat{H}=3.0, a_{\mathrm{w}}=0.2$ and $\beta_{\mathrm{b}}=0.91$, (b) $\varepsilon=0.01, \hat{H}=3.0$ and $\beta_{\mathrm{b}}=0.93$. 
The plot of the resonance curves corresponding to Eq. (36) by setting $\alpha_{\mathrm{i}}=0$ and $n=-1,-2,-3$ are illustrated in Fig. 4. The dashed lines show group-I and group-II orbits, which are represented in Fig. 1. The Kolmogorov-Arnold-Moser (KAM) theory states that if the system becomes nonintegrable due to small perturbation of Hamiltonian, the invariant tori will be typically deformed but not destroyed. We know that one of the conditions that must be satisfied for KAM theorem is that the torus is non-resonant. Chaos can occur when KAM theorem does not hold [11]. As we see in this analysis that resonance occurs and this implies that in this system, under the special initial condition, we can see the chaos. Analysis of self-field induced resonance shows that this chaoticity originates from the coupling between the guide-field induced betatron oscillation and the helical

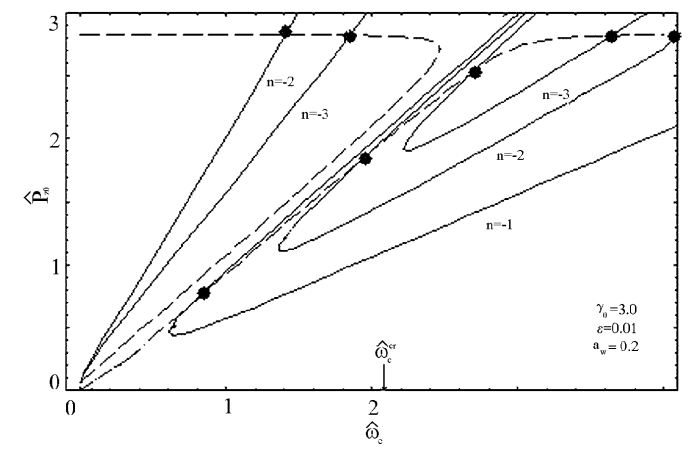

Fig. 4. Graph of the equilibrium self-field resonance curves (solid curves) corresponding to the solutions to Eq. (36) for $n=-1,-2,-3, \varepsilon=0.01, \alpha_{\mathrm{i}}=0, \gamma_{0}=3.0$, and $a_{\mathrm{w}}=0.2$. The dashed curves are the integrable steady-state orbits calculated in Fig. 1, and dots signify the intersections between the resonance curves and the steady-state orbits.

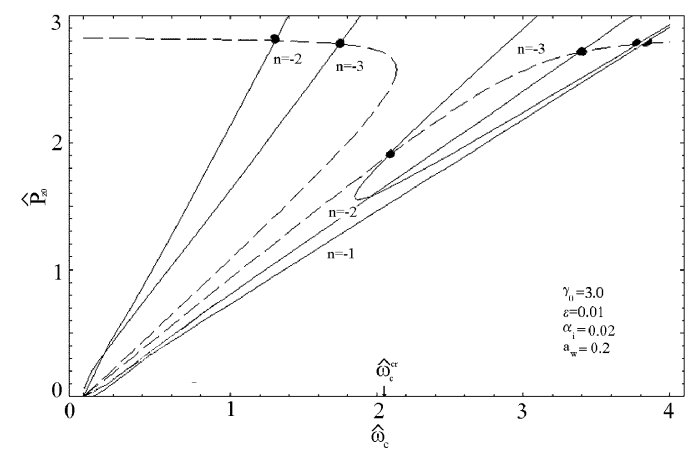

Fig. 5. Graph of the equilibrium self-field resonance curves (solid curves) corresponding to the solutions to Eq. (36) for $n=-1,-2,-3, \varepsilon=0.01, \alpha_{\mathrm{i}}=0.02, \gamma_{0}=3.0$, and $a_{\mathrm{w}}=0.2$. The dashed curves are the integrable steady-state orbits calculated in Fig. 1, and dots signify the intersections between the resonance curves and the steady-state orbits. 
motion modified by radial gradient of the self-field effect. Strong chaotic behavior is caused by the overlap of the self-field induced resonance. There is a good agreement between computer simulation and analytic estimate. The second-order island appearing near the group-II orbits in Fig. 3b occurs near the intersection between the $n=-2$ resonance curves and the group-II orbits at $\omega_{\mathrm{c}}=4$ in Fig. 5 . But our purpose is to illustrate the effect of ion-channel guiding on chaos. For this objective we plot resonance curves for Eq. $(36)\left(\alpha_{\mathrm{i}} \neq 0\right)$ and $n=-1,-2,-3$. It is clear from Fig. 5 that the coupling, causing chaos, does not occur by the effect of the ion-channel guiding. From this in Fig. 5 we do not have any intersection between the resonance curve and group-II orbits at $\omega_{\mathrm{c}}=4$. This means that we do not have chaos and ion-channel guiding effect removes the chaos effect. Poincaré surface of section maps for this case are illustrated for two cases of group I and group II. The value of the dimensionless parameters in Fig. 6 is $(\mathrm{a}) \varepsilon=0.01, \hat{\omega}_{\mathrm{c}}=2.0, \hat{H}=3.0$, $a_{\mathrm{w}}=0.2, \beta_{\mathrm{b}}=0.91$, and $\alpha_{\mathrm{i}}=0.02$, (b) $\varepsilon=0.01, \hat{\omega}_{\mathrm{c}}=4.0, \hat{H}=3.0, a_{\mathrm{w}}=0.2$, $\beta_{\mathrm{b}}=0.93$ and $\alpha_{\mathrm{i}}=0.02$. As we see, Figs. 6a and b are in agreement with Fig. 5 .
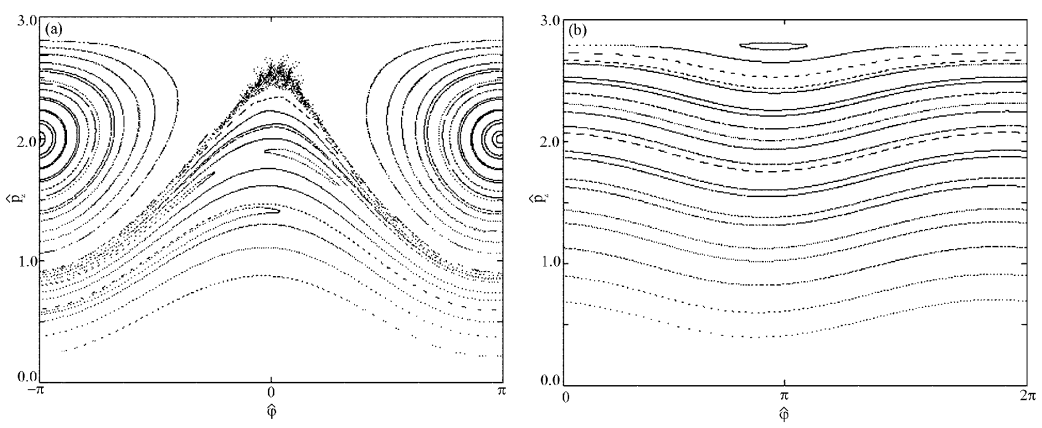

Fig. 6. Graph of the modified electron trajectories in presence of ion-channel guiding, for the two cases (a) $0<\hat{\omega}_{\mathrm{c}}=2.0<\hat{\omega}_{\mathrm{cr}} \cong 2.1$ and (b) $\omega_{\mathrm{c}}=4.0>\omega_{\mathrm{cr}}$. Other system parameters are (a) $\varepsilon=0.01, \hat{H}=3.0, a_{\mathrm{w}}=0.2, \alpha_{\mathrm{i}}=0.02$ and $\beta_{\mathrm{b}}=0.91$, (b) $\varepsilon=0.01, \alpha_{\mathrm{i}}=0.02, a_{\mathrm{w}}=0.2, \hat{H}=3.0$, and $\beta_{\mathrm{b}}=0.93$.
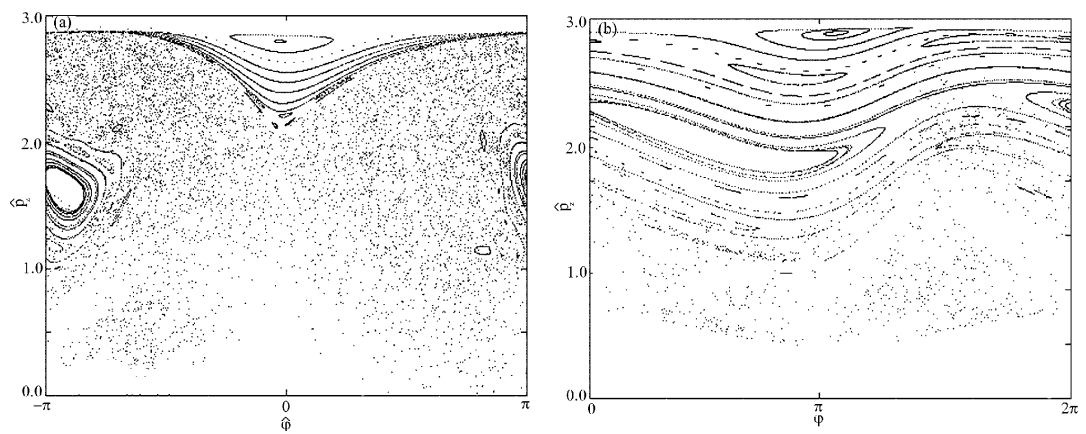

Fig. 7. Graph of nonintegrable surface-of-section for two cases (a) $0<\hat{\omega}_{\mathrm{c}}=2.0<\hat{\omega}_{\mathrm{cr}}$ and (b) $\omega_{\mathrm{c}}=4.0>\omega_{\mathrm{cr}}$. Other system parameters are (a) $\varepsilon=0.026, \hat{H}=3.0, a_{\mathrm{w}}=0.2$ and $\beta_{\mathrm{b}}=0.91$ and $(\mathrm{b}) \varepsilon=0.026, \hat{H}=3.0$ and $\beta_{\mathrm{b}}=0.93$. 

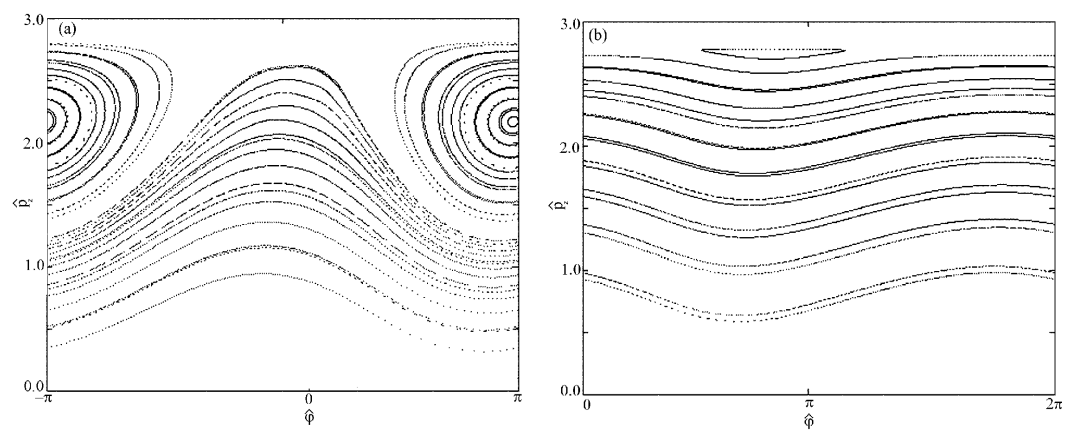

Fig. 8. Graph of the modified electron trajectories in presence of ion-channel guiding for two cases (a) $0<\hat{\omega}_{\mathrm{c}}=2.0<\hat{\omega}_{\mathrm{cr}} \cong 2.1$ and (b) $\omega_{\mathrm{c}}=4.0>\omega_{\mathrm{cr}}$. Other system parameters are (a) $\varepsilon=0.026, \hat{H}=3.0, a_{\mathrm{w}}=0.2, \alpha_{\mathrm{i}}=0.045$ and $a_{\mathrm{w}}=0.2, \beta_{\mathrm{b}}=0.91$, (b) $\varepsilon=0.026, \alpha_{\mathrm{i}}=0.045, a_{\mathrm{w}}=0.2, \hat{H}=3.0$ and $\beta_{\mathrm{b}}=0.93$.

Comparison of Figs. 6 and 3 shows that the ion-channel guiding contribution, Fig. 6, modifies the chaotic motion arising from self-field effects shown in Fig. 3. In Figs. 7 and 8 we investigate the effects of a change in $\varepsilon$ and $\alpha_{\mathrm{i}}$ in the chaotic motion. First, we set $\alpha_{\mathrm{i}}=0$ and $\varepsilon=0.026$, the nonintegrable motions are shown in Fig. 7a for group I and Fig. 7b for group II. Second, to modify this nonintegrable motion we increase the parameter $\alpha_{\mathrm{i}}$ of ion-channel guiding i.e., $\alpha_{\mathrm{i}}=0.045$, as shown in Fig. 8a for group I and Fig. 8b for group II. Therefore an increase in $\varepsilon$ requires an increase in $\alpha_{\mathrm{i}}$ to keep the nonintegrable motion of self-field effect modified.

\section{References}

[1] H.P. Freund, J.M. Antonsen, Principle of Free-Electron-Lasers, 2nd ed., Chapman and Hall, London 1996.

[2] R.C. Davidson, H.S. Uhm, Phys. Fluids 23, 2070 (1980).

[3] H. Mehdian, M. Esmelzadeh, J.E. Willet, Phys. Plasmas 8, 3776 (2001).

[4] K.T. Akayama, S. Hiramatsu, Phys. Rev. A 37, 173 (1988).

[5] P. Jha, P. Kumar, IEEE Trans. Plasma Sci. 24, 359 (1996).

[6] P. Jha, P. Kumar, Phys. Rev. E 57, 2256 (1998).

[7] R.C. Davidson, Theory of Non-neutral Plasmas, Addison Wesley, Reading, MA 1989.

[8] C. Chen, R.C. Davidson, Phys. Fluids B 2, 171 (1989).

[9] T.J. Orzechowski, B.R. Anderson, J.C. Clark, W.M. Fawley, A.C. Paul, D. Prosnitz, E.T. Scharlemann, S.M. Yarema, D.B. Hopkins, A.M. Sessler, J.S. Wurtele, Phys. Rev. Lett. 57, 2172 (1986).

[10] J. Masud, J.C. Marshall, S.P. Schlesinger, F.G. Yee, Phys. Rev. Lett. 56, 1567 (1986).

[11] S. Jorna, Topics in Nonlinear Dynamics a Tribute to Edward Bullard, American Institute of Physics, New York 1978. 